\title{
Avaliação de tratamentos endodônticos realizados por alunos de graduação
}

\author{
Evaluation of endodontic treatments performed by hiring student \\ Evaluación de tratamientos de endodoncia realizados por estudiantes
}

\section{Resumo}

O controle clínico-radiográfico, pós-tratamento endodôntico, permite avaliar e comprovar os resultados da terapia endodôntica, classificando-a como sucesso ou fracasso. O sucesso endodôntico pode ser definido como o resultado da terapia endodôntica, quando o dente clinicamente apresenta-se assintomático, está funcional e sem patologia radiográficat. Este estudo teve como objetivo avaliar a qualidade do tratamento endodôntico realizados por alunos de graduação da Faculdade de Odontologia da Universidade de Pernambuco, Brasil. Os prontuários odontológicos da amostra incluíram todos os pacientes atendidos em 2000 e 2022. Os testes Qui- quadrado e Exato de Fisher foram utilizados para calcular o nível de significância estabelecido em 5\%. O tamanho da amostra foi de 219 pacientes e foi possível verificar o sucesso na maioria dos tratamentos, observando-se um percentual de $85,4 \%$ (188 pacientes) e o insucesso em apenas 31 pacientes $(14,2)$. Com relação ao limite apical da obturação do sistema de canais radiculares, que o sucesso do tratamento foi bem maior quando a obturação estava no forame apical e no CDC, 92.0 e 91,4\%, respectivamente e o pior resultado em casos de sub-obturação, 70,0\%, com diferença estatisticamente significativa. Conclui-se que existiu um percentual elevado de sucesso do tratamento endodôntico realizados por alunos de graduação da Faculdade de Odontologia de Pernambuco indicando que os acadêmicos estão recebendo um ensino de qualidade.

Palavras-chave: Endodontia; Tratamento do canal radicular; Obturação do canal radicular.

\begin{abstract}
The clinical-radiographic control, after endodontic treatment, allows to evaluate and prove the results of endodontic therapy, classifying it as success or failure. Endodontic success can be defined as the final result of endodontic therapy, when the tooth is clinically asymptomatic, functional and without radiographic pathology. This study aimed to evaluate the quality of endodontic treatment performed by undergraduate students at the Faculty of Dentistry, University of Pernambuco, Brazil. The dental records of the sample included all patients seen in 2000 and 2022 . Chisquare and Fisher's exact tests were used to calculate the significance level set at 5\%. The sample size was 219 patients and it was possible to verify the success in most treatments, observing a percentage of $85.4 \%$ (188 patients) and failure in only 31 patients (14.2). Regarding the apical limit of the root canal system obturation, the treatment success was much higher when the obturation was in the apical foramen and in the CDC, 92.0 and $91.4 \%$, respectively and the worst result in cases of sub-obturation , 70.0\%, with a statistically significant difference. It is concluded that there was a high percentage of success in endodontic treatment performed by undergraduate students at the Faculty of Dentistry of Pernambuco, indicating that academics are receiving quality education.
\end{abstract}

Keywords: Endodontics; Root canal therapy; Root canal obturation. 


\begin{abstract}
Resumen
El control clínico-radiográfico, posterior al tratamiento endodóntico, permite evaluar y comprobar los resultados de la terapia endodóntica, clasificándola como éxito o fracaso. El éxito endodóntico se puede definir como el resultado final de la terapia endodóntica, cuando el diente se encuentra clínicamente asintomático, funcional y sin patología radiográfica. Este estudio tuvo como objetivo evaluar la calidad del tratamiento de endodoncia realizado por estudiantes de pregrado en la Facultad de Odontología de la Universidad de Pernambuco, Brasil. Los registros dentales de la muestra incluyeron a todos los pacientes atendidos en 2000 y 2022. Se utilizaron las pruebas de chicuadrado y exacta de Fisher para calcular el nivel de significancia establecido en 5\%. El tamaño de la muestra fue de 219 pacientes y se pudo comprobar el éxito en la mayoría de los tratamientos observándose el porcentaje del 85,4\% (188 pacientes) y el fracaso en solo 31 pacientes $(14,2)$. En cuanto al límite apical de la obturación del sistema de conductos radiculares, el éxito del tratamiento fue mucho mayor cuando la obturación fue en el foramen apical y en el CDC, 92,0 y 91,4\%, respectivamente y el peor resultado en casos de sub-obturación, 70,0\%, con una diferencia estadísticamente significativa. Se concluye que hubo un alto porcentaje de éxito en el tratamiento de endodoncia realizado por estudiantes de graduación de la Facultad de Odontología de Pernambuco, lo que indica que los académicos están recibiendo una formación de calidad.
\end{abstract}

Palabras clave: Endodoncia; Tratamiento del conducto radicularo; Obturación del conducto radicular.

\title{
1. Introdução
}

O profissional precisa convencer-se de que o tratamento endodôntico não termina com a obturação do sistema de canais radiculares, visto que a longo prazo, a qualidade da reparação periapical deve ser almejada, uma vez que o tratamento endodôntico não se limita tecnicamente ao exclusivo preenchimento de um espaço preparado, mas também a um período de controle clínico-radiográfico pós-tratamento endodôntico.

Entende-se como o preparo químico-mecânico dos canais radiculares uma das etapas mais importantes no tratamento endodôntico (Amaral et al. 2019), seu objetivo é realizar a limpeza e desinfecção, eliminando irritantes microbianos que são as principais causas da periodontite apical (Siqueira \& Lopes 2011). Assim, podemos afirmar que o sucesso do tratamento endodôntico depende da limpeza e modelagem do canal radicular (Paque et al. 2009). Além disso, a cinemática e o número de instrumentos utilizados no tratamento também podem influenciar a qualidade final do preparo do canal. (Tavares et al. 2019).

A determinação da qualidade, por sua vez, é realizada através do exame clínico, o exame radiográfico e a análise histopatológica. O profissional dispõe como recursos o controle longitudinal, baseando-se unicamente nas características clínicas (sinais e sintomas) e nos aspectos radiográficos. Os resultados do tratamento dos canais radiculares têm sido avaliados em diversas pesquisas epidemiológicas,seja através de estudos transversais ou estudos longitudinais. O sucesso é dependente de diversos fatores pré-operatórios, assim como dos resultados do preparo e da obturação dos canais radiculares e por ocasionais contratempos no tratamento. Parece que os dentes tratados com polpas vitais têm melhor prognóstico do que aqueles com polpas necróticas (Travassos, et al. 2002)

Alguns aspectos geram dúvidas na avaliação de controle dos tratamentos endodônticos, dentre eles, a ausência de sinais e sintomas endodônticos associados à parcial reparação das áreas radiolúcidas que é considerado tratamento bemsucedido por Carvalho et al, 2019. Entretanto para Travassos, et al, 2021, essa condição só seria considerada quando da ocorrência da completa reparação das estruturas periapicais, radiograficamente, combinada à ausência de sinais e sintomas endodônticos.

Portanto, torna-se difícil confrontar alguns resultados, ora devido às diferentes metodologias empregadas, ora pela inerente dificuldade em classificar os inúmeros estágios radiográficos periapicais de controle, observados nos períodos póstratamento endodônticos.

O período de acompanhamento pós-tratamento endodôntico também varia nas diversas pesquisas; alguns autores acreditam que 1 ano seria um período suficiente para avaliar o sucesso, enquanto outros requerem um tempo mínimo de 4 a 5 anos (Yasmim, et. al, 2021) afirmam com muita propriedade que mesmo após 2 anos de concluído a terapia endodôntica, pode existir uma diminuição da radiolucência radiográfica da lesão. Estas variações entre os diversos trabalhos devem ser levadas 
em consideração, assim como, o tamanho da amostra, tratamentos endodônticos realizados por alunos de graduação, pósgraduação, clínicos gerais, docentes e até ospróprios pesquisadores em suas clínicas particulares.

Por estes motivos, a presente pesquisa foi realizada com o intuito dereconhecer os fatores relacionados aos sucessos dos tratamentos endodônticos executados por alunos de graduação e, dessa forma, identificando também os fatores que influenciam no insucesso com a finalidade de se chegar ao aprimoramento da previsibilidade da terapia endodôntica realizada.

\section{Metodologia}

O presente estudo foi aprovado pelo comitê de ética e pesquisa da Faculdade de Odontologia da Universidade de Pernambuco (FOP-UPE), datada de 04 de outubro de 2000, conforme Meno PROPEGE/CPQ/CE n 28/2000, Protocolo ${ }^{\circ}$ 026/00, Parecer $n^{\circ}$ E026/00. Foram selecionados prontuários de 219 pacientes com canais radiculares tratados nas Clínicas de Atenção Básica II e III entre os anos de 2000 a 2004. A importância de fazer a proservação de um tratamento endodôntico, com o intuito de confirmar a qualidade do atendimento no nosso serviço. Foi realizado um estudo de coorte retrospectivo de avaliação dos tratamentos endodônticos realizados por alunos da Faculdade de Odontologia, Universidade de Pernambuco. O sucesso clínico foi indicado pela ausência de sinais e sintomas e assumiu-se que o quadro clínico o sucesso pode ter uma forte relação com o sucesso radiográfico determinado pelos seguintes critérios: 1) nenhuma lesão periapical ou lesão em andamento presente no tempo de obturação; 2) lesão periapical presente no momento de obturação desapareceu completamente ou foi visivelmente diminuído de tamanho.

Os pacientes que completaram o tratamento nos períodos mencionados foram chamados para comparecerem à Faculdade para a realização dos exames clínico e radiográfico de controle. Para que os achados radiográficos fossem adequadamente analisados na ocasião do exame de controle, todas as radiografias realizadas durante o tratamento endodôntico deviam ser de boa qualidade, com o mínimo de distorção, especialmente as radiografias inicial e final do caso. Os critérios adotados nesta pesquisa para avaliar o sucesso ou o fracasso do tratamento endodôntico foram baseados nos critérios e radiográficos conforme o quadro abaixo:

Quadro 1. Critérios adotados na pesquisa.

\begin{tabular}{|l|c|c|c|}
\hline \multicolumn{1}{|r|}{ RADIOGRÁFICO } & SUCESSO & INSUCESSO & QUESTIONÁVEL \\
\hline CLINIICO & SUCESSO & INSUCESSO & QUESTIONÁVEL \\
\hline SUCESSO & QUESTIONÁVEL & INSUCESSO & QUESTIONÁVEL \\
\hline QUESTIONÁVEL & INSUCESSO & INSUCESSO & INSUCESSO \\
\hline INSUCESSO & INO
\end{tabular}

Fonte: Autores.

\section{Resultados}

O teste Qui-quadrado de independência foi utilizado para verificar a presença ou não de associação significativa para cada uma das variáveis independentes com a variável resposta avaliação do tratamento endodôntico, sendo também apresentado o valor do Odds Ratio (OR). Ressalta-se que nas situações em que o teste Qui- quadrado não foi verificado, utilizou-se o teste Exato de Fisher para as tabelas de dimensão 2 x 2 ou o teste da Razão de Verossimilhança para as tabelas de 
dimensão superior a 2 x 2. O nível de significância considerado nos testes estatísticos foi de 5,0\%. Os “softwares” estatísticos utilizados para a obtenção dos cálculos estatísticos foram o SAS (Statistical Analysis System), na versão 6.12, e o SPSS, na versão 8 .

Em relação à Tabela 1, é possível verificar que a maioria dos tratamentos foi avaliada como sucesso, observando-se um percentual de $85,4 \%$ (188 pacientes) e o insucesso em apenas 31 pacientes $(14,2)$.

Tabela 1 - Avaliação do sucesso da terapia endodôntica.

\begin{tabular}{l|c|c|l|l}
\hline Avaliação do tratamento & \multicolumn{2}{|l|}{} & \multicolumn{2}{c}{ OR e IC com 95,0\% } \\
\hline & $\mathrm{N}$ & $\%$ & & \\
\hline Sucesso & & & & \\
\hline & 188 & 85,8 & $\mathrm{P}=0,898$ & $1,03(0,59$ a 1,82) \\
\hline Incucesso & & & & 1,00 \\
\hline & 31 & 14,2 & & \\
\hline TOTAL & & & & \\
\hline & & & & \\
\hline
\end{tabular}

Em relação à Tabela 1, não existiu diferença estatística significativa, no sucesso do tratamento endodôntico realizados por alunos de graduação da Faculdade de Odontologia de Pernambuco, 85,8\% quando comparado ao insucesso da terapia $(14,2 \%)$.

Tabela 2 - Avaliação do tratamento endodôntico de acordo com o limite de obturação.

\begin{tabular}{|c|c|c|c|c|c|c|c|}
\hline \multirow{3}{*}{ Variáveis } & \multicolumn{4}{|c|}{ Avaliação do tratamento } & & & \multirow{3}{*}{ Valor de P } \\
\hline & \multicolumn{2}{|c|}{ Sucesso } & \multicolumn{2}{|c|}{ Insucesso } & \multicolumn{2}{|c|}{ TOTAL } & \\
\hline & $\mathrm{N}$ & $\%$ & $\mathrm{~N}$ & $\%$ & $\mathrm{~N}$ & $\%$ & \\
\hline \multicolumn{8}{|c|}{$\begin{array}{l}\text { - Limite de obturação } \\
\text { do canal radicular }\end{array}$} \\
\hline $\mathrm{CDC}$ & 138 & 91,4 & 13 & 8,6 & 151 & 100,0 & $\mathrm{P}^{(1)}<0,001^{*}$ \\
\hline Obturação exata & 23 & 92,0 & 2 & 8,0 & 25 & 100,0 & \\
\hline Sub-obturação & 21 & 70,0 & 9 & 30,0 & 30 & 100,0 & \\
\hline Sobre-obturação & 6 & 85,7 & 1 & 14,3 & 7 & 100,0 & \\
\hline \multirow[t]{2}{*}{ Dente extraído } & - & - & 6 & 100,0 & 6 & 100,0 & \\
\hline & 188 & 85,8 & 31 & 14,2 & 219 & 100,0 & \\
\hline
\end{tabular}

- -Diferença significativa ao nível de 5. Fonte: Autores.

Nessa tabela, observa-se que o sucesso do tratamento foi bem maior, com relação ao limite apical da obturação do sistema de canais radiculares quando estavam no forame apical e no CDC, 92.0 e 91,4\%, respectivamente e o pior resultado em casos de sub-obturação, 70,0\%, com diferença estatisticamente significativa. 
Tabela 3 - Avaliação do tratamento endodôntico de acordo com preenchimento da obturação.

\begin{tabular}{|c|c|c|c|c|c|c|c|c|c|}
\hline $\begin{array}{l}\text { - Preenchimento do } \\
\text { canal radicular }\end{array}$ & & & & & & & & & \\
\hline Com falha & 30 & 83,3 & 6 & 16,7 & 36 & 100,0 & 30 & 83,3 & $\mathrm{P}<0,001^{*}$ \\
\hline Sem falha & 158 & 89,3 & 19 & 10,7 & 177 & $\begin{array}{c}100 \\
0\end{array}$ & 158 & 89,3 & \\
\hline Dente extraído & - & - & 6 & $\begin{array}{c}100 \\
0\end{array}$ & 6 & $\begin{array}{c}100 \\
0\end{array}$ & - & - & \\
\hline & 30 & 83,3 & 6 & 16,7 & 36 & $\begin{array}{c}100 \\
0\end{array}$ & 30 & 83,3 & \\
\hline
\end{tabular}

* - Associação significativa ao nível de 5,0\%. Fonte: Autores.

Com relação à Tabela 3, observa-se que existiu diferença estatística significativa, determinando maior percentual de sucesso, $89,3 \%$ quando não existia falha na obturação e um menor com a presença de falha no preenchimento do canal radicular $(83,3 \%)$.

\section{Discussão}

A avaliação do reparo de uma lesão periapical, com o objetivo de controlar a terapia endodôntica, através da variável radiográfica, é utilizada nos estudos prospectivos e retrospectivos, sejam eles clínicos e radiográficos ou apenas radiográficos, bem como nos estudos do tipo: ensaios clínicos, série de casos, estudo de casos. Essa variável é adotada para classificar o tratamento endodôntico em sucesso, questionável ou insucesso, e em algumas metodologias, apenas em sucesso ou insucesso. Bem como o período de proservação que está na dependência da variável utilizada para investigar o tratamento endodôntico. Os estudos que avaliam clinicamente, observam apenas a condição dolorosa ocorrida no pós-operatório imediato e, consequentemente, esse período fica reduzido a até 48 horas após determinados procedimentos endodônticos de até 30 dias. As pesquisas que avaliam clínica e radiograficamente ou apenas pela variável radiográfica, analisam o pós-operatório tardio, e esse tempo está na dependência do tempo mínimo ou máximo necessário que os pesquisadores adotam para avaliar a resolução de uma área radiolúcida periapical. o sucesso endodôntico pode ser definido como o resultado final da terapia endodôntica, quando o dente apresenta-se clinicamente assintomático, funcionalmente ativo e sem patologia radiográfica. Não obstante, o exame radiográfico apenas sugere informações e deve ser considerado dentro de um parâmetro temporal Castro, et. al, 2020. Dessa forma, o sucesso de um tratamento endodôntico depende de um acompanhamento, realizando=se um controle clínico e radiográfico do paciente, verificando alguns critérios clínicos e radiográficos, como: dor, edema, fístula, presença ou aumento de lesão periapical. Se todas as etapas do tratamento endodôntico forem realizadas adequadamente, espera-se que, após o período de 1 a 2 anos de avaliação, o sucesso seja alcançado. (Alves-Silva, 2021). Deve-se ressaltar que sendo um estudo retrospectivo, não foi possível padronizar os fatores geométricos e energéticos utilizados durante a exposição radiográfica, nem o processamento do filme (Travassos et al., 2003). Diante de tais aspectos, os examinadores nesta pesquisa foram orientados para interpretarem as radiografias inicial e de controle, uma vez que é obrigatório a utilização de posicionador radiográfico nesses momentos, para a análise do limite longitudinal da obturação do canal radicular e qualidade do preenchimento do canal radicular (densidade do material obturador e adaptação do material obturador às paredes do canal radicular).

O controle clínico-radiográfico, pós-tratamento endodôntico, permite avaliar e comprovar os resultados da terapia endodôntica, classificando-a como sucesso ou fracasso. O sucesso endodôntico pode ser definido como o resultado final da terapia endodôntica, quando o dente clinicamente apresenta-se assintomático, está funcional e sem patologia radiográfica (Oliveira et al., 2018). Entretanto, o insucesso pode estar relacionado com o desenvolvimento de rarefações periapicais depois da terapia realizada, isto é, onde não existia nenhuma alteração óssea anterior ao tratamento, como também com a persistência 
ou aumento do tamanho da lesão periapical tomada como referência, Travassos, Albuquerque, Caldas Júnior, Santos, 2005. Convém salientar que muitas vezes a lesão periapical demora a regredir após a terapêutica, e o controle radiográfico deverá ser realizado um ano após o tratamento (Travassos et al., 2003). Sendo assim, a avaliação clínica e radiográfica dos resultados obtidos com o tratamento do canal radicular, é uma conduta clássica, apesar de as pesquisas terem demonstrado diferentes percentuais de sucesso ou insucesso.

Os critérios clínicos são de fundamental importância para classificar o tratamento endodôntico como sucesso ou insucesso, visto que, se esse estudo fosse apenas radiográfico, muitos fatores que influenciam negativamente a terapia endodôntica não poderia ser reproduzida. Em função desses problemas, essa pesquisa analisou situações clínicas e radiográficas, valorizando-se muito o exame clínico do paciente, desde a presença de dor, fístula, mobilidade, presença bolsa periodontal. Com relação às exodontias, as pesquisas que não consideram os dentes extraídos na sua amostra, provavelmente, aumentando o percentual de sucesso e assim existirá um viés metodológico pois esses dentes em estudos retrospectivos deveriam ser incluídos na análise do insucesso da terapia endodôntica, portanto, nesta pesquisa que encontrou 6 dentes extraídos que foram classificados como insucesso.

Concernente às evidências de reparo de uma área radiotransparência óssea, não existe um critério definido. Na maior parte dos estudos, a persistência e/ ou o aumento da rarefação óssea periapical, bem como o aparecimento de uma lesão periapical onde anteriormente não existia é categorizada como fracasso da reparação óssea (Oliveira et al., 2018), A redução da lesão periapical, talvez seja a mais polêmica de todas, já que alguns pesquisadores a categorizam como questionável e outros como sucesso ou fracasso. Os estudos de Vojinovic et al. (2010), mostram que o período de 6 meses não é suficiente para obter uma imagem radiográfica clara da recuperação de todos os tecidos periodontais, e que o ideal seria 12 meses após a finalização do tratamento endodôntico. Para alguns pesquisadores, uma lesão periapical diminuída é classificada como fracasso, mesmo que o dente esteja assintomático (Benenati \& Khajotia 2002, Travassos et al., 2005). De forma contrária, diversos trabalhos classificam uma lesão diminuída como questionável, que resulta em um menor padrão de fracasso (Travassos et al. 2021). No entretanto, alguns pesquisadores classificam a diminuição da lesão periapical como sucesso do tratamento endodôntico (Travassos \& Negreiros, 2021, Yasmim, et al, 20201), demonstraram que os exames clínicos e radiográficos de controle apresentaram resultados altamente satisfatórios, com resolução completa de todos os sinais e sintomas clínicos e significativa diminuição da radiolucência radiográfica da lesão após anos de controle. Por esses motivos, neste estudo, preferiu-se classificar como questionável, já que existem lesões periapicais que necessitam de 4 a 5 anos para ocorrer o reparo total da lesão periapical.

Os tratamentos endodônticos foram analisados, também, com relação a fatores que podem influenciar negativamente no resultado da avaliação de controle do tratamento, dentre eles, o limite longitudinal da obturação do canal radicular; o preenchimento do canal radicular (densidade do material obturador e adaptação do material obturador às paredes do canal radicular). Os padrões de sucesso variam entre 50 e 95\% (Travassos \& Negreiros, 2021). A variação deve-se a diversos aspectos, dentre os quais estão: as diferenças nos critérios aplicados para determinar o sucesso e, portanto, pelas experiências do conhecimento especializado das pessoas que realizaram os tratamentos. No presente estudo, os tratamentos endodônticos realizados por alunos de graduação da Faculdade de Odontologia de Pernambuco alcançaram o patamar de sucesso de 85,8\%, constatando-se, um aumento ainda maior quando relacionado ao limite apical da obturação do sistema de canais radiculares quando estavam no forame apical e no CDC, 92.0 e 91,4\%, respectivamente. Diante de um insucesso do tratamento endodôntico, o clínico deve tentar estabelecer a possível causa do problema. Algumas vezes, a origem pode ser facilmente identificada, porém em muitos casos não há evidência de uma causa. No estudo, observou-se 70,0\% de insucesso nos casos de sub-obturação, existindo uma diferença estatisticamente significativa, corroborando com Pinheiro, et al. (1998) que comprovaram que a inadequada obturação dos canais radiculares está associada a uma maior incidência de lesões periapicias, 
uma vez que dos 173 canais radiculares qualificados como mal obturados, 131 eram portadores de lesões periapicais. Em relação à extensão apical da obturação, constataram que a presença de lesão no periápice apresentou maior incidência as subobturações (75.81\%) Discordando de Souza (1998), que afirma que o limite apical de obturação não é a etapa mais importante para a obtenção do sucesso endodôntico. Esse está diretamente associado à qualidade da limpeza e modelagem do canal radicular, incluindo a patência do forame apical, remoção da camada residual e medicação intracanal. Contudo, o índice de sucesso endodôntico realizados por alunos de graduação da Faculdade de Odontologia de Pernambuco apresentado pelo estudo foi elevado, uma vez que, foi possível avaliar a regressão total de uma lesão periapical com proservação clínica e radiográfica em apenas dois anos.

\section{Conclusão}

Conclui-se que existiu um percentual elevado de sucesso do tratamento endodôntico realizados por alunos de graduação da Faculdade de Odontologia de Pernambuco indicando que os acadêmicos estão recebendo um ensino de qualidade.

\section{Referências}

Alves-Silva, E. G., de Medeiros, J. B. G., de Medeiros, W. D., Dametto, F. R., Arruda-Vasconcelos, R., Louzada, L. M., \& Gadê-Neto, C. R. (2021). Insertos ultrassônicos na desobstrução de canais com pinos de fibra de vidro: estudo in vitro. Research, Society and Development, $10(2)$, e3481029536-e3481029536.

Alves-Silva, E. G., Paiva, A. C. T. S., Rêgo, L. G., Arruda-Vasoncelos, R., Louzada, L. M., de Almeida Gomes, B. P. F., \& de Carvalho, L. K. C. G. (2021). Proservação de tratamentos endodônticos realizados na clínica odontológica. Research, Society and Development, 10(11), e532101119724-e532101119724.

Alves-Silva, E. G., Souza, P. X., Solano, N. T., Carvalho, R. A., Dametto, F. R., de Almeida Gomes, B. P. F., \& Carvalho, L. K. C. G. (2020). Análise da qualidade das radiografias executadas durante os tratamentos endodônticos realizados na graduação da Universidade Potiguar. Archives of health investigation, $9(3)$.

Amaral, R. R., Oliveira, A. G. G., Braga, T., Reher, P., de Macêdo Farias, L., Magalhães, P. P., \& de Souza Côrtes, M. I. (2020). Quantitative assessment of the efficacy of two different single-file systems in reducing the bacterial load in oval-shaped canals: a clinical study. Journal of Endodontics, 46(9), 12281234.

Arruda-Vasconcelos, R., Louzada, L. M., Feres, M., Tomson, P. L., Cooper, P. R., \& Gomes, B. P. F. A. (2021). Investigation of microbial profile, levels of endotoxin and lipoteichoic acid in teeth with symptomatic irreversible pulpitis: a clinical study. International Endodontic Journal, 54(1), 46-60.

Barbosa, I. O., \& Travassos, R. M. C. (2021). Regressão de lesão periapical. Revista FAIPE, 10(2), 49-55.

Benenati, F. W., \& Khajotia, S. S. (2002). A radiographic recall evaluation of 894 endodontic cases treated in a dental school setting. Journal of endodontics, 28(5), 391-395.

Carvalho, M. C., Zuolo, M. L., Arruda-Vasconcelos, R., Marinho, A. C. S., Louzada, L. M., Francisco, P. A., ... \& Gomes, B. P. F. D. A. (2019). Effectiveness of XP-Endo Finisher in the reduction of bacterial load in oval-shaped root canals. Brazilian oral research, 33.

Castro, L. R., Lara, N. C., Reis, I. P., Ribeiro, I. F., Swerts, A. A., \& Tavares, É. P. (2020). Reparo periapical em tratamentos endodônticos com extravasamento de cimento obturador-relato de caso. Revista Científica da UNIFENAS, 2(2).

Ferreira, G. D. S., Travassos, R. M. C., Schmitz, M. D. S., \& Melo, Â. (2007). Verificação da concordância inter e intra-examinadores no contole radiográfico de lesões periapicais. $R F O U P F, 37-41$.,

Oliveira, N. G., de Vasconcelos Carvalho, M., \& Travassos, R. M. C. (2018). Regressão de lesão periapical extensa: relato de caso clínico. Revista de Odontologia da Universidade Cidade de São Paulo, 30(2), 210-215.

Paqué, F., Ganahl, D., \& Peters, O. A. (2009). Effects of root canal preparation on apical geometry assessed by micro-computed tomography. Journal of endodontics, 35(7), 1056-1059.

Siqueira JF Jr, Lopes HP. Chemomechanical preparation. In: Siqueira JF Jr, ed. Treatment of Endodontic Infections. London: Quintessence Publishing; 2011: 236-84

Tavares, S. J. D. O., Sarmento, E. B., Guimaraes, L. D. S., Antunes, L. A. A., Antunes, L. S., \& Gomes, C. C. (2019). The influence of kinematics of enginedriven nickel-titanium instruments on root canal shape assessed by micro-computed tomography: a systematic review. Acta Odontologica Scandinavica, 77(5), 347-358.

Travassos, R. M. C., Albuquerque, D. S. D., Caldas Junior, A. D. F., \& Santos, R. A. D. (2005). Avaliação da terapia endodôntica. Odontol. clín.-cient, 189192. 
Research, Society and Development, v. 11, n. 3, e16711326336, 2022

(CC BY 4.0) | ISSN 2525-3409 | DOI: http://dx.doi.org/10.33448/rsd-v11i3.26336

Travassos, R. M. C., de Oliveira, A. C. C., dos Passos Gomes Filho, C. H., da Silva Sousa, I. S., da Silva Alves, J. M., dos Santos, K. M., \& do Prado, V. F. F. (2021). Análise de regressão da lesão periapical: relato de caso clínico. Research, Society and Development, 10(12), e201101220267-e201101220267.

Travassos, R. M. C., Negreiros, J. H. C. N., de Araújo Teixeira, J., Lyra, M. C. A., Barbosa, L. M., \& Netto, O. J. R. L. (2021). Tratamento endodôntico conservador em lesão periapical extensa asséptica: Relato de caso. Research, Society and Development, 10(5), e33710514982-e33710514982.

Vojinović, J., Čupić, S., Dolić, O., Mirjanić, Đ., Sukara, S., \& Obradović, M. (2010). Success rate of the endodontic treatment of young permanent teeth with calcium hydroxide. growth, 4,8 .

Yamin, P. A., Fernandes, A. M., da Silva Botega, L., Mori, A. A., \& Júnior, A. A. J. Tratamento de lesão cística periapical com reabsorção externa: relato de caso e proservação de 2 anos. Odonto, 29(56), 17-25.

Zoti, M., \& Hartmann, M. S. M. (2016). Avaliação de tratamentos endodônticos realizados por alunos de graduação da Escola de Odontologia da IMED. Journal of Oral Investigations, 5(1), 4-12. 